\title{
Clay nanosheet-mediated delivery of recombinant plasmids expressing artificial miRNAs via leaf spray to prevent infection by plant DNA viruses
}

Qili Liu (1) ${ }^{1,2,3}$, Yanpeng $\mathrm{Li}^{4}$, Kedong $\mathrm{Xu}^{5}$, Dongxiao $\mathrm{Li}^{3}$, Haiyan $\mathrm{Hu}^{3}$, Feng Zhou ${ }^{3}$, Puwen Song ${ }^{3}$, Yongang $\mathrm{Yu}^{3}$, Qichao Wei ${ }^{3}$, Qian Liư ${ }^{1}$, Weipeng Wang ${ }^{1}$, Ruifang Bu ${ }^{3}$, Haili Sun ${ }^{3}$, Xiaohui Wang ${ }^{4}$, Jianjun $\mathrm{HaO}^{6}$, Honglian $\mathrm{Li}^{2}$ and Chengwei $\mathrm{Li}^{1,3,5}$

\begin{abstract}
Whitefly-transmitted begomoviruses are economically important plant pathogens that cause severe problems in many crop plants, such as tomato, papaya, cotton, and tobacco. Tomato yellow leaf curl virus (TYLCV) is a typical monopartite begomovirus that has been extensively studied, but methods that can efficiently control begomoviruses are still scarce. In this study, we combined artificial microRNA (amiRNA)-mediated silencing technology and clay nanosheetmediated delivery by spraying and developed a method for efficiently preventing TYLCV infection in tomato plants. We designed three amiRNAs that target different regions of TYLCV to silence virus-produced transcripts. Three plant expression vectors expressing pre-amiRNAs were constructed, and recombinant plasmid DNAs (pDNAs) were loaded onto nontoxic and degradable layered double hydroxide (LDH) clay nanosheets. $\mathrm{LDH}$ nanosheets containing multiple pDNAs were sprayed onto plant leaves. We found that the designed amiRNAs were significantly accumulated in leaves 7 days after spraying, while the pDNAs were sustainably detected for 35 days after the spray, suggesting that the LDH nanosheets released pDNAs in a sustained manner, protected pDNAs from degradation and efficiently delivered pDNAs into plant cells. Importantly, when the LDH nanosheets coated with pDNAs were sprayed onto plants infected by TYLCV, both the disease severity and TYLCV viral concentration in sprayed plants were significantly decreased during the 35 days, while the levels of $\mathrm{H}_{2} \mathrm{O}_{2}$ were significantly increased in those plants. Taken together, these results indicate that LDH nanosheets loaded with pDNAs expressing amiRNAs can be a sustainable and promising tool for begomovirus control.
\end{abstract}

\section{Introduction}

Whitefly-transmitted begomoviruses are economically important plant pathogens that cause severe problems in many crop plants, such as tomato, papaya, cotton, and tobacco ${ }^{1}$. Tomato yellow leaf curl virus (TYLCV) is one of the most important viral pathogens of tomatoes. It belongs to the genus Begomovirus and is distributed

\footnotetext{
Correspondence: Xiaohui Wang (fewangxh@scut.edu.cn) or Honglian Li (honglianli@sina.com) or Chengwei Li (lichengweiwau@hotmail.com)

${ }^{1}$ Postdoctoral Research Base, Henan Institute of Science and Technology, Xinxiang, China

${ }^{2}$ College of Plant Protection, Henan Agricultural University, Zhengzhou, Henan, China

Full list of author information is available at the end of the article
}

worldwide, being the 3rd most important plant virus ${ }^{1,2}$. Begomoviruses have circular and single-stranded DNA molecules encapsulated in a twinned (quasi)-icosahedral virion ${ }^{2}$. They are classified into two groups based on their genome organization: monopartite, having a single genome component, and bipartite, having two genome components, DNA-A and DNA- $\mathrm{B}^{3}$. DNA-A and DNA-B are $2.7-2.8 \mathrm{~kb}$ in size, and each component has its own open reading frames (ORFs) oriented in a bidirectional fashion. Monopartite begomoviruses (or DNA-A of bipartite begomoviruses) have six ORFs, with two in the virion sense orientation (AV1/V1 and AV2/V2, encoding the coat protein $(\mathrm{CP})$ and the V2/AV2 protein,

\section{(c) The Author(s) 2020}

(c) (i) Open Access This article is licensed under a Creative Commons Attribution 4.0 International License, which permits use, sharing, adaptation, distribution and reproduction cc) in any medium or format, as long as you give appropriate credit to the original author(s) and the source, provide a link to the Creative Commons license, and indicate if changes were made. The images or other third party material in this article are included in the article's Creative Commons license, unless indicated otherwise in a credit line to the material. If material is not included in the article's Creative Commons license and your intended use is not permitted by statutory regulation or exceeds the permitted use, you will need to obtain permission directly from the copyright holder. To view a copy of this license, visit http://creativecommons.org/licenses/by/4.0/. 
respectively) and four in the complementary orientation (AC1/C1-AC4/C4, encoding the replication associated protein (Rep), or $\mathrm{C} 1 / \mathrm{AC} 1$ protein; the transcriptional activator protein (TrAP), or $\mathrm{C} 2 / \mathrm{AC} 2$ protein; the replication enhancer protein (REn), or $\mathrm{C} 3 / \mathrm{AC} 3$ protein; and the C4/AC4 protein) $)^{3}$ TYLCV is a monopartite begomovirus.

Managing plant viruses is difficult because no chemicals are highly effective in suppressing viral infection in plants. In recent years, interest in gene silencing mediated by artificial microRNAs (amiRNAs) has increased ${ }^{4-7}$. In this technology, an amiRNA is designed to target genes responsible for viral replication, transmission, or symptom development and interferes with the multiplication and spread of viruses in host plants ${ }^{4}$. amiRNAs are singlestranded ( $21 \mathrm{nt}$ long) and are designed by replacing a mature microRNA (miRNA) sequence with a duplex structure within pre-miRNAs ${ }^{4}$. Owing to certain attributes, such as their uniqueness, effectiveness and precision, gene silencing through amiRNAs is considered a second-generation technology of RNA interference (RNAi) $^{5}$. It is easy to optimize single amiRNA sequences to target one or several mRNAs without disturbing the expression of other genes ${ }^{6,7}$.

Delivery of genetic materials and regeneration of transformed plants are very important for genetic transformation of plants, the latter being highly impacted by the liability and stability of the delivery method ${ }^{8,9}$. Methods such as electroporation, biolistics, agrobacterium-mediated delivery, and cationic delivery typically require the regeneration of genetically modified progeny plants. Furthermore, agrobacterium-mediated delivery introduces another pathogen into plants. These methods are not perfect and need improvement in applications ${ }^{10-13}$. Therefore, there is a need to develop more effective, simple, and economical methods.

To enhance the stability, translation efficiency, and cell targeting of a naked mRNA, nanomaterials have been used as effective transportation tools in the genetic engineering of plants ${ }^{14-16}$. Particularly, they have been applied for delivering amiRNAs into plants ${ }^{17-19}$. In this way, dsRNAs can be transferred into plant leaves ${ }^{20}$, $\operatorname{roots}^{21,22}$ and flower buds ${ }^{23}$. Mitter et al. reported that a single spray of dsRNA loaded on a designed, nontoxic, and degradable layered double hydroxide (LDH) (BioClay) can protect plants against pepper mild mottle virus (PMMoV) and cucumber mosaic virus (CMV), which greatly prolongs the protection of plant leaves from 7 to 20 days $^{20}$. dsRNA can enter plants and has a slow release of the RNAs carried with LDH delivery ${ }^{20}$. Recombinant DNA plasmids (pDNAs) are often used in plant genetic engineering, but the delivery of pDNA is an important element affecting the efficiency of plant transformation. The larger and sterically hindered pDNA brings new challenges for the loading and delivery of biomolecules with LDH. The aims of this study were to, for the first time, use LDH nanosheets as a delivery system for transporting recombinant pDNAs into plants and examine the effect of TYLCV suppression in plants.

\section{Results}

amiRNA design and preparation of pDNA-LDH nanosheets

To improve amiRNA-mediated resistance in plants against TYLCV, three TYLCV-specific amiRNAs were designed based on the conserved region of the AV1 gene and a partial region of the AV2 gene. The designed amiRNAs were amiR-AV1/AV2-1, amiR-AV1-2, and amiR-AV1-3, which targeted three different regions of the AV1 gene (Table S1). AmiR-AV1/AV2-1 targeted the $5^{\prime}$ end of the AV1 transcript and the middle region of the AV2 transcript, as these two genes overlap each other with two different reading frames. AmiR-AV1-2 and amiR-AV1-3 targeted the middle and downstream regions of the AV1 transcript, respectively. No potential off-target effects of these amiRNAs were observed within the available tomato genome database. All three preamiRNAs (Table S3) were detected in the cloned plasmid pBI121 using PCR and sequencing (Fig. S1). The structures of the pre-miR159a backbone were predicted, including those for pre-amiRNA-1, pre-amiRNA-2, and pre-amiRNA-3 (Fig. S2).

To understand how LDH supported pDNA on a pDNA-LDH nanosheet, both LDH and pDNA-LDH were analyzed. LDH nanoparticles, which are made up of positively charged brucite-like layers, were synthesized by a microwave-assisted aqueous coprecipitation method. Energy-dispersive X-ray spectrometry (EDS) analysis gave an approximate chemical formula of $\operatorname{Mg} 16.01 \mathrm{Al} 6.44(\mathrm{OH})$ $12.78\left(\mathrm{NO}_{3}\right) 2.07\left(\mathrm{CO}_{3}\right) 11.69 \cdot 9.73 \mathrm{H}_{2} \mathrm{O}$ (Fig. S3). As measured by dynamic light scattering, the LDH nanoparticles possessed a positive zeta potential of $+44.9 \mathrm{mV}$. The $\mathrm{LDH}$ nanoparticles exhibited a narrow size distribution with an average hydrodynamic diameter of $35.43 \pm 0.07 \mathrm{~nm}$ (Zaverage particle size) and polydispersity index (PDI) of 0.24 , which were much smaller than those of LDHs prepared by hydrothermal treatment $(102.56 \pm 1.01 \mathrm{~nm}$, with a PDI of 0.16), suggesting that microwave-assisted treatment is a promising way to reduce the preparation time and maintain the necessary morphology.

X-ray diffraction and Fourier transform infrared (FTIR) spectrum analyses of the collected nanomaterials showed typical features of MgAl-LDH-type materials. The X-ray diffraction patterns of LDH samples (Fig. S4a) displayed a series of sharp peaks from basal diffractions at $10.7^{\circ}, 22^{\circ}$, and $34^{\circ}$ corresponding to planes (003), (006), and (009), indicating good crystallinity in the typical layered structure. The basal spacing $(0.82 \mathrm{~nm})$ was slightly larger than that reported in the literature, which indicated a greater 
chance of interlayer ion exchange. Based upon the full width at half maximum at the (003) plane and the Scherrer equation, the thickness of the pristine LDH nanoparticles was calculated to be $6.08 \mathrm{~nm}$. FTIR spectra of LDH samples displayed characteristic absorption bands at 3486 and $1646 \mathrm{~cm}^{-1}$, which were attributed to stretching vibrations of $\mathrm{O}-\mathrm{H}$ and bending vibrations of hydroxyl groups of the interlayered water molecules (Fig. S4b). The peak at $673 \mathrm{~cm}^{-1}$ was assigned to metal-oxygen-metal and metal-O-H stretching. Similar to previous observations, the absorption band at $1376 \mathrm{~cm}^{-1}$ was the asymmetric stretching vibration mode of $\mathrm{C}-\mathrm{O}$ in carbonate, which overlapped the signal of the stretching vibration of $\mathrm{NO}_{3}{ }^{-}$. The morphological features of LDH samples were characterized by scanning electron microscopy (SEM) and transmission electron microscopy (TEM). SEM images showed that the LDH nanoparticles had lateral dimensions varying from 30 to $60 \mathrm{~nm}$ while maintaining a narrowly distinguished polygonal plateletlike nanosheet morphology (Fig. S4c). Most sheets were well formed in a hexagonal shape (Fig. S4d, e).

Successful formation of a pDNA-LDH complex was confirmed by negligible migration from wells during electrophoresis (Fig. 1a). The constructed pDNA pBI121amiR showed complete loading at pDNA:LDH mass ratios of 1:10 (pBI121-amiR-AV1/AV2-1 and pBI121-amiRAV1-2, Fig. 1a lane 7) and 1:12 (pBI121-amiR-AV1-3, Fig. 1a lane 8). This indicated that LDH nanosheets bound purified pDNA molecules $\sim 13 \mathrm{~kb}$ in length. Compared to pristine LDH nanomaterials (Fig. 1b), pDNA-LDH composites were larger (average $\sim 80 \mathrm{~nm}$ ), while the surface of pDNA-LDH composites were smoother, and the nanoparticles mostly showed a round shape (Fig. 1c). A part of the structure of pDNA was also observed between LDH nanoparticles (Fig. 1c).

\section{Stability of pDNAs on LDH nanosheets}

For pDNA-LDH to provide prolonged pDNA-mediated protection against viruses, pDNA must not only remain on the leaf surface for a longer period than naked pDNA but also withstand field conditions, such as rain, sunlight, and nucleases. Atmospheric $\mathrm{CO}_{2}$ and moisture can slowly breakdown LDH into biocompatible residue $\left(\mathrm{Mg}^{2+}, \mathrm{Al}\right.$ $(\mathrm{OH})_{3}$, and $\mathrm{NO}^{3-}$, and so on), releasing the loaded biomolecules $^{24}$. To evaluate the delivery and release of pDNA bonded on LDH nanosheets, the stability of pDNA-LDH was examined for delivering and releasing pDNA in acidic conditions. The $\mathrm{pH}$ increased from acidic to basic values within $60 \mathrm{~min}$ from the introduction of diluted nitric acid ( $\mathrm{pH}$ 3.0) to LDH solution. The initial

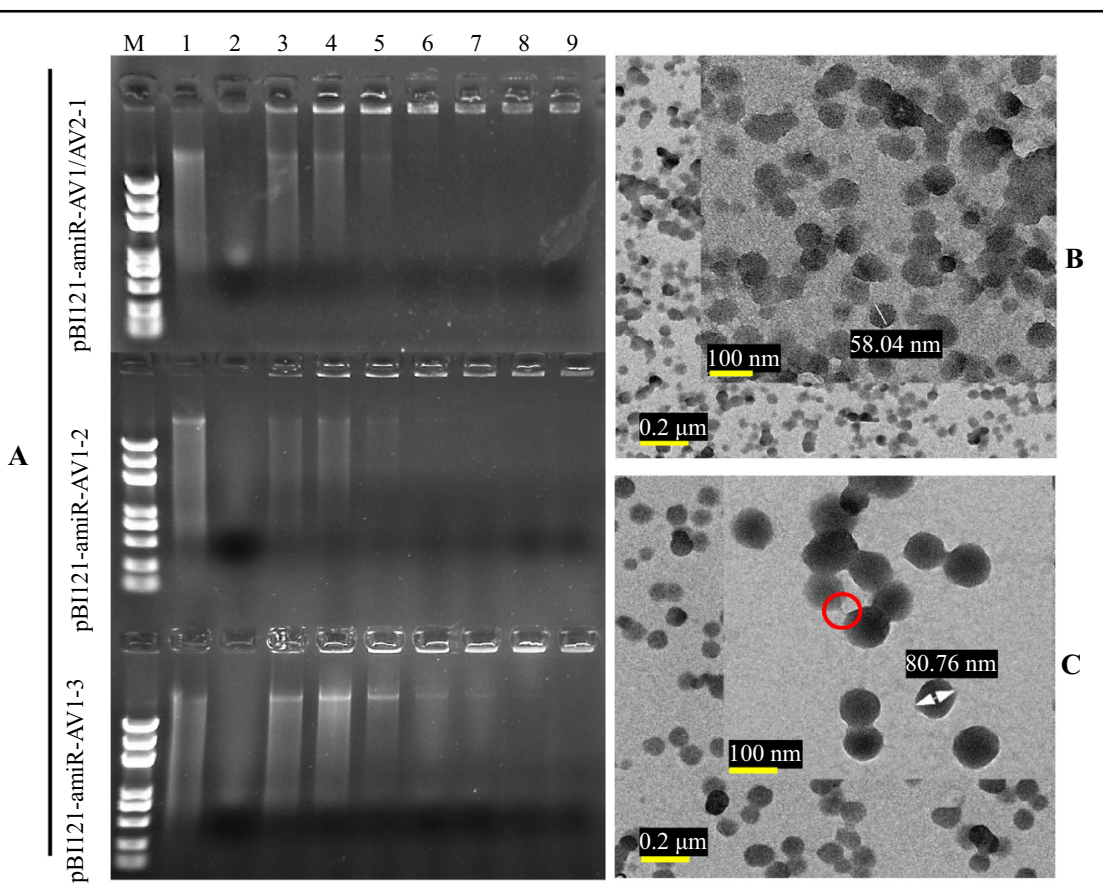

Fig. 1 Characterization of layered double hydroxide (LDH) nanosheets and loaded pDNA. a pDNA at pDNA:LDH mass ratios of 1:1, 1:2, 1:4, 1:8, 1:10, 1:12, and 1:16, corresponding to lanes 3, 4, 5, 6, 7, 8, and 9, respectively. pDNA only (lane 1) and LDH only (lane 2). M =5 kb DNA ladder, LDHbound pDNA was detected by fluorescence in wells. Complete loading was achieved at pDNA-LDH mass ratios of 1:10 (lane 7) and 1:12 (lane 8). b Transmission electron microscopy (TEM) images of the LDH structure. c TEM images of the pDNA-LDH nanocomposite. The surface of the pDNA-LDH composites was smooth, and the nanoparticles tended to have a round shape. The red circle shows a part of the structure of pDNA between LDH nanosheets. Dose ratio $=1: 1, T=298 \mathrm{~K}$ 


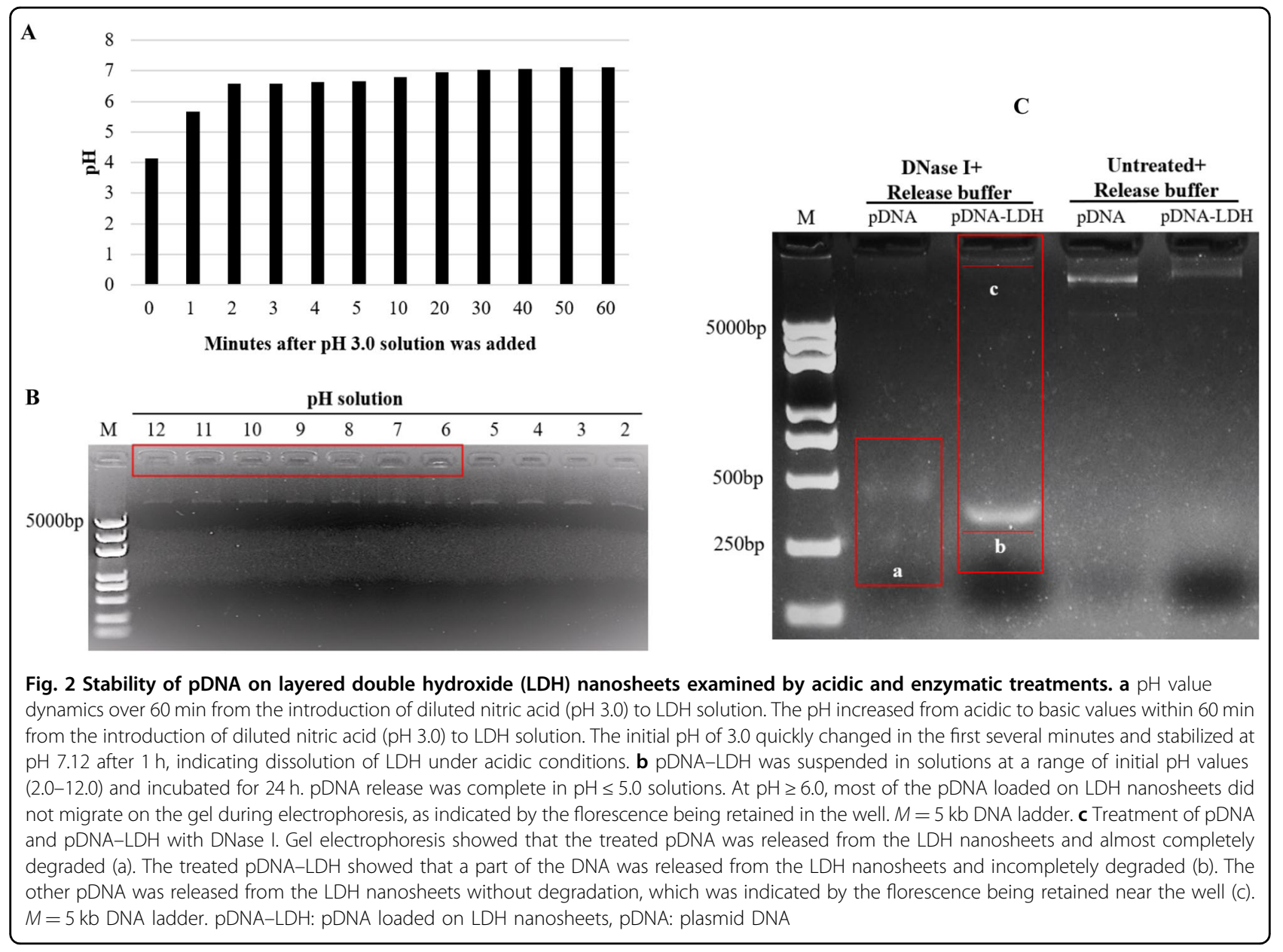

$\mathrm{pH}$ of 3.0 quickly changed in the first several minutes and stabilized at $\mathrm{pH} 7.12$ after $1 \mathrm{~h}$, indicating dissolution of LDH under acidic conditions (Fig. 2a). pDNA-LDH was resuspended in solutions at a range of initial $\mathrm{pH}$ values (2.0-12.0) and incubated for $24 \mathrm{~h}$. Complete release of pDNA was observed at $\mathrm{pH} 2.0,3.0,4.0$, and 5.0, which was reflected by no florescence being retained in the well (Fig. 2b). At $\mathrm{pH} \geq 6.0$, most of the pDNA loaded on LDH nanosheets did not migrate on the gel during electrophoresis, as indicated by the florescence being retained in the well (Fig. 2b).

The ability of the LDH nanosheets to protect loaded pDNA from potential environmental breakdown, such as enzymatic conditions, was investigated by exposure of pDNA and pDNA-LDH to DNase I. Gel electrophoresis showed that the treated pDNA was released from the $\mathrm{LDH}$ nanosheets and almost completely degraded (Fig. 2c-a). The treated pDNA-LDH showed that some of the DNA was released from the LDH nanosheets and incompletely degraded (Fig. 2c-b), while the other pDNA was released from the LDH nanosheets without degradation, which was indicated by the florescence being retained near the well (Fig. 2c-c). Accordingly, DNase I treatment degraded the naked pDNA to a greater extent than the pDNA bonded to LDH nanosheets, suggesting that the LDH nanosheets protected the pDNAs from DNase I degradation.

\section{Delivery of pDNAs into plant cells and amiRNA expression}

To examine the efficiency of foliar application of pDNA-LDH, YOYO-1 was used to label the nanosheets for visualization in plant cells. Once the pDNA/YOYO-1 complexes were formed, they were stable and exhibited a very high affinity between dye and the pDNA. The results indicated that cells of onion epidermis and Nicotiana benthamiana leaves treated with YOYO-pDNA-LDH showed an abundance of YOYO in the nucleus or membranes after $72 \mathrm{~h}$ post treatment (Fig. 3).

Systemic movement of YOYO florescence occurred from the outside to the inside of the plant cells in the YOYO-pDNA-LDH-treated plants. These observations suggested that pDNA can be taken up by plant cells with the help of LDH nanosheet transport and release. Moreover, at 35 days after spraying, the pBI121 vector was 


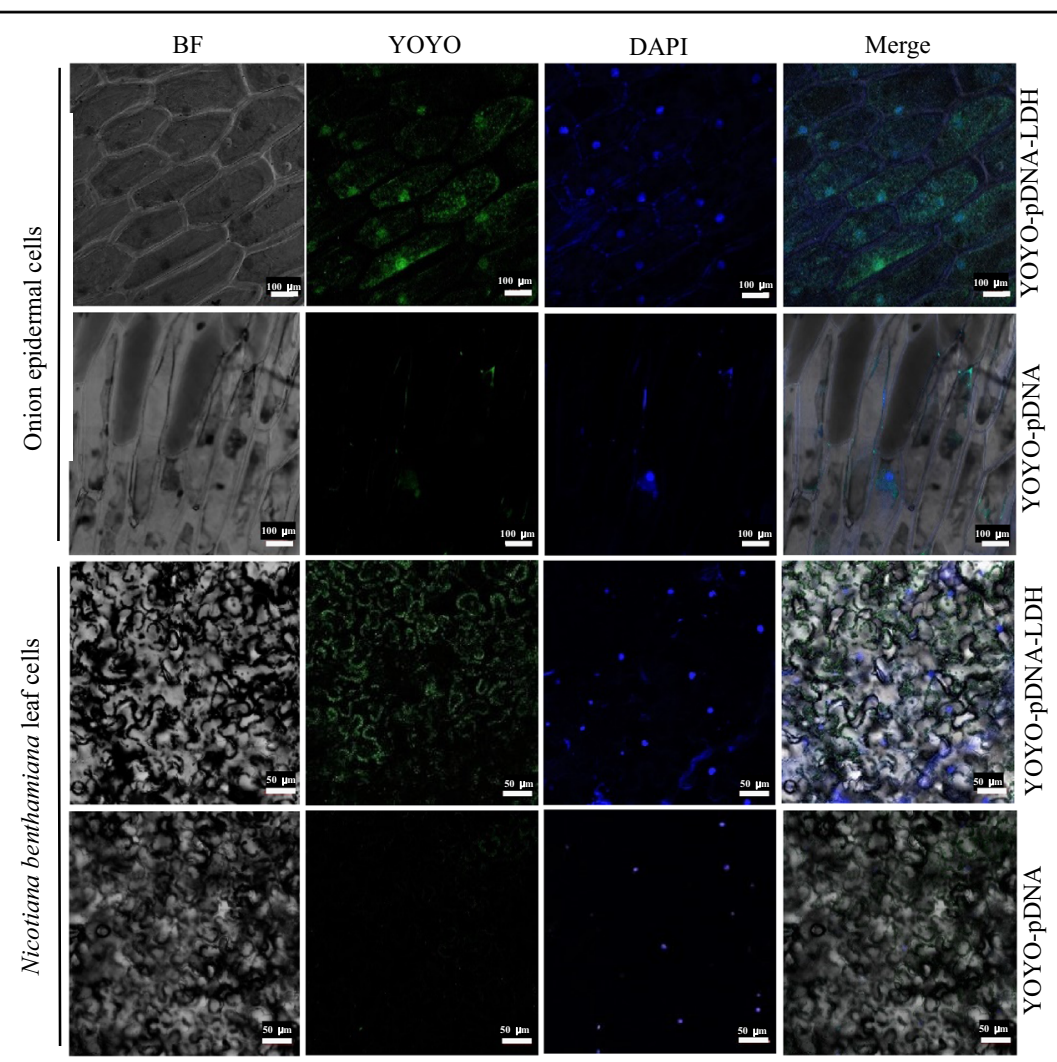

Fig. 3 The delivery efficiency of foliar application of pDNA-LDH into plant cells. Cells of onion epidermis and Nicotiana benthamiana leaves were observed under a confocal microscope, with bright field (BF), YOYO florescence, DAPI stain and merged images shown for samples at $72 \mathrm{~h}$ after treatment with YOYO-pDNA-LDH and YOYO-pDNA. YOYO-pDNA-LDH: pDNA loaded on LDH nanosheets and labeled with YOYO, YOYO-pDNA: pDNA labeled with YOYO

detected from nearby unsprayed new leaves, which indicated that the pDNA-LDH was delivered systemically from sprayed leaves to unsprayed new leaves in the treated N. benthamiana and Solanum lycopersicum plants (Fig. S5).

Expression of mixed mature amiRNAs was characterized by qRT-PCR using a Mir- $\mathrm{X}^{\mathrm{TM}}$ miRNA First-Strand Synthesis kit (TaKaRa Bio, USA) for diverse treatments of $N$. benthamiana and S. lycopersicum to investigate their role in suppressing TYLCV. In N. benthamiana treated with multiple pDNA-LDHs, amiRNA expression was upregulated to levels approximately seventy times that of the control group and greater than those of the other single pDNA-LDH treatments (Fig. 4a). In S. lycopersicum treated with multiple pDNA-LDHs, amiRNA expression was approximately one hundred and ninety times that of the control group (Fig. 4b).

\section{Preventing TYLCV by foliar spray of pDNA-LDH}

Thirty minutes after foliar spraying, leaf cells and stomata of $N$. benthamiana and S. lycopersicum plants were not significantly different between pDNA-LDH, pDNA, and control treatments (Fig. S6). At 35 days post-TYLCV inoculation, the infectivity of TYLCV in N. benthamiana and S. lycopersicum plants harboring amiRNA was significantly suppressed compared to those of plants receiving the other treatments (Table S2). Disease incidence and severity were both lower in mixed pDNA-LDH-treated plants than in nontreated plants (Fig. 5a). All plants inoculated with TYLCV showed viral symptoms that spread from the top of the young leaves but the appearance times of symptoms in mixed pDNA-LDH-treated plants were significantly delayed (Fig. 5a). The symptom expression rate of mixed pDNA-LDH-treated S. lycopersicum plants was $41.7 \%$ (Table S2) over up to 35 days after inoculation, which was significantly lower than that for other treatments and closely related to the high expression of mixed mature amiRNAs (Fig. 4).

To determine the effect of mixed pDNA-LDH application on TYLCV prevention, the disease severity, accumulation of $\mathrm{H}_{2} \mathrm{O}_{2}$, and viral concentrations in TYLCV-infected $N$. benthamiana and S. lycopersicum were measured. $\mathrm{H}_{2} \mathrm{O}_{2}$ significantly increased in the leaves of the plants treated with pDNA-LDH compared to other treated plants (Fig. 5b). These results suggest that mixed 

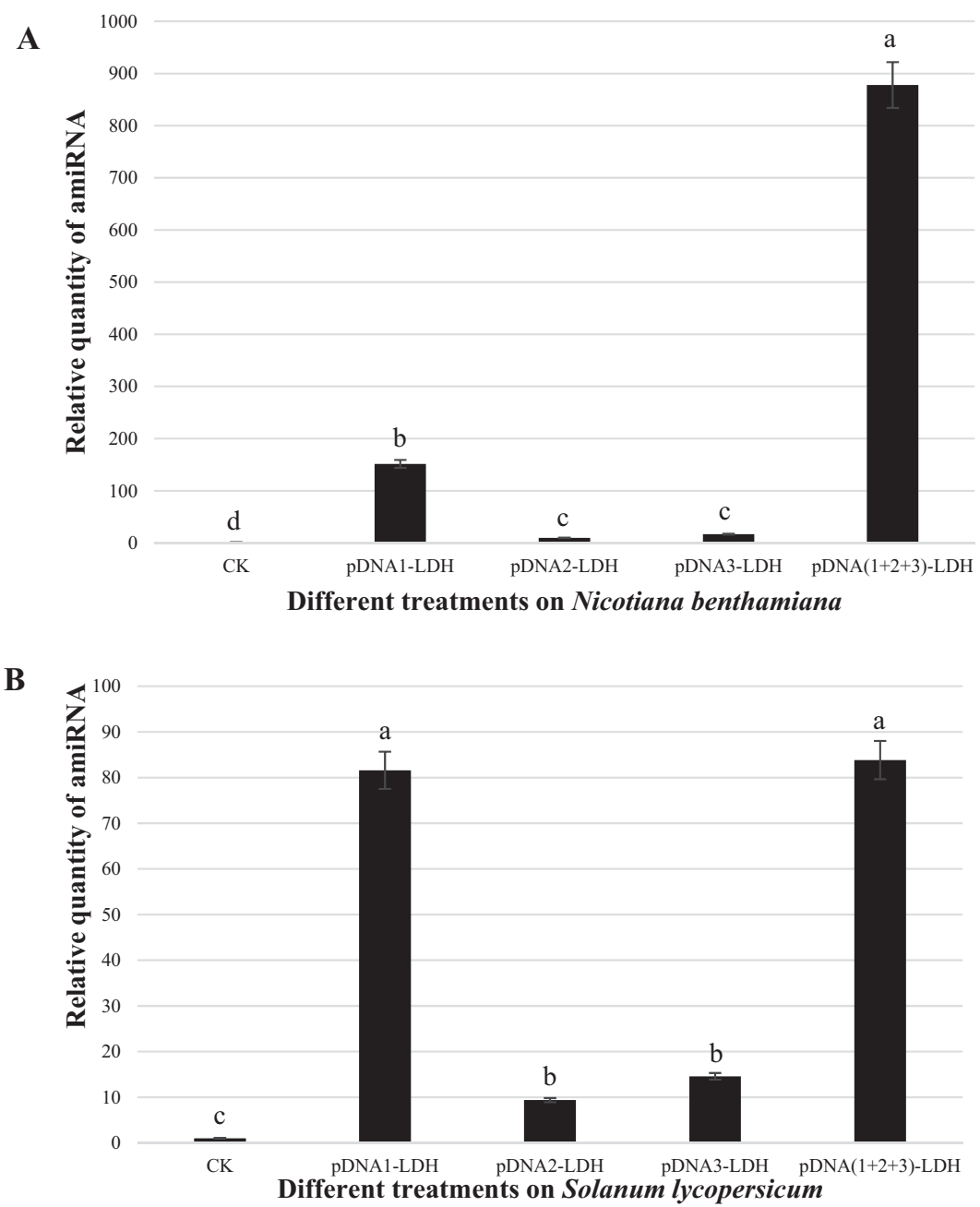

Fig. 4 Expression of mixed mature amiRNAs for diverse treatments. Expression levels of mixed mature miRNAs in Nicotiana benthamiana (a) and Solanum lycopersicum (b) after they were sprayed with pBI121-LDH (CK), pDNA1-LDH (amiRNA-AV1/AV2-1), pDNA2-LDH (amiRNA-AV1-2), pDNA3-LDH (amiRNA-AV1-3), and pDNA(1 + 2 + 3)-LDH (mixed with three pDNA-LDH), as determined by qRT-PCR assays. The bars for each column represent standard deviations. Values (means \pm SEs) are the averages of three independent experiments, and significant differences $(P \leq 0.05)$ among treatments in each group are indicated by different letters

pDNA-LDH application effectively protected S. lycopersicum against TYLCV for at least the 35-day period. Mixed pDNA-LDH treatment significantly reduced the concentration of TYLCV in the leaves of $N$. benthamiana and S. lycopersicum (Fig. 6a, b).

\section{Discussion}

We have demonstrated that plasmids carrying amiRNAs were successfully delivered into plant cells with the help of LDH nanosheets and that the amiRNAs formed in the transformed plant cells were effective in suppressing the replication of TYLCV and symptom expression. The expression of amiRNA in S. lycopersicum leaves treated with multiple LDH-carrying pDNAs was significantly upregulated at 35 days post-TYLCV inoculation. In addition, by using multiple amiRNAs targeting different regions of the CP gene of TYLCV, the strategy reduced the risk of pathogens overcoming RNAi. LDH nanosheets were proven to be an effective carrier and delivery system for pDNA. More importantly, their slow release extended the period of effectiveness and assured long-lasting protection of plants against viral infection.

The stability of pDNA on LDH nanosheets was the key to effectively protecting and releasing pDNAs into different locations of plant cells. LDH nanosheets have been studied for carrying small RNAs and efficiently delivering them to mammalian cells in vitro ${ }^{25}$ and for protecting and releasing large double-stranded RNAs into plant cells ${ }^{20,26}$. Genomic DNA fragments of $\sim 500-1000 \mathrm{bp}^{27}$, PCR products of $800 \mathrm{bp}^{28}$ and DNA molecules of $8 \mathrm{~kb}^{29}$ can be 


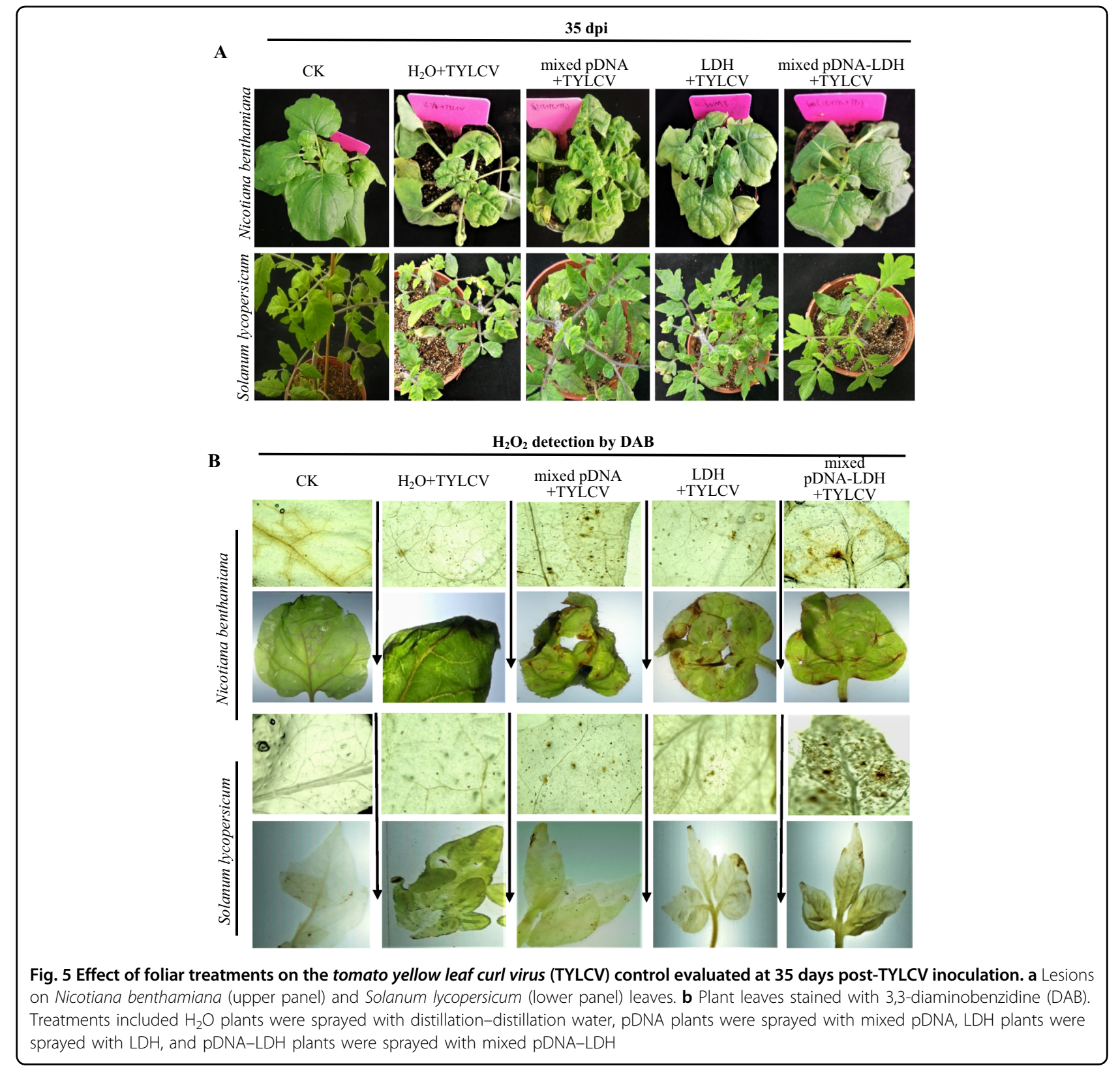

intercalated in the interlayer galleries of LDHs by anion exchange or a direct self-assembly route and can then be delivered into animal cells. It seems that large and sterically hindered biomolecules such as pDNAs usually adopt a supercoiled structure in solution and do not fully intercalate in the interlayer galleries by means of anion exchange $^{30}$. In this study, we also observed a small amount of incomplete intercalation of pDNA into LDH sheets, which should not impede cellular uptake and/or protection from degradation, which is in agreement with results from Ladewig ${ }^{30}$.

In delivering transgenic materials into plants, pDNA has some advantages over $\mathrm{RNA}^{20}$. High-quality pDNA is the key to successful genetic transformation in plants. pDNA can not only be propagated in Escherichia coli (E. coli) but also be produced in large quantities through fermentation. Methods for the rapid production of large amounts of pDNA include bioprocessing, boiling ${ }^{31}$, a modified boiling method $^{32}$ and alkaline lysis. Alkaline lysis is the most widely used method for extracting pDNAs from $E$. $\operatorname{coli}^{33,34}$. To achieve alkaline lysis at a large scale for the extraction of pDNAs from E. coli, Wright et al. used a simple stirred tank reactor system and increased the pDNA quantity very quickly by sequentially adding three precipitants, including a selective precipitation step with ammonium acetate ${ }^{34}$. This pDNA could be incorporated 


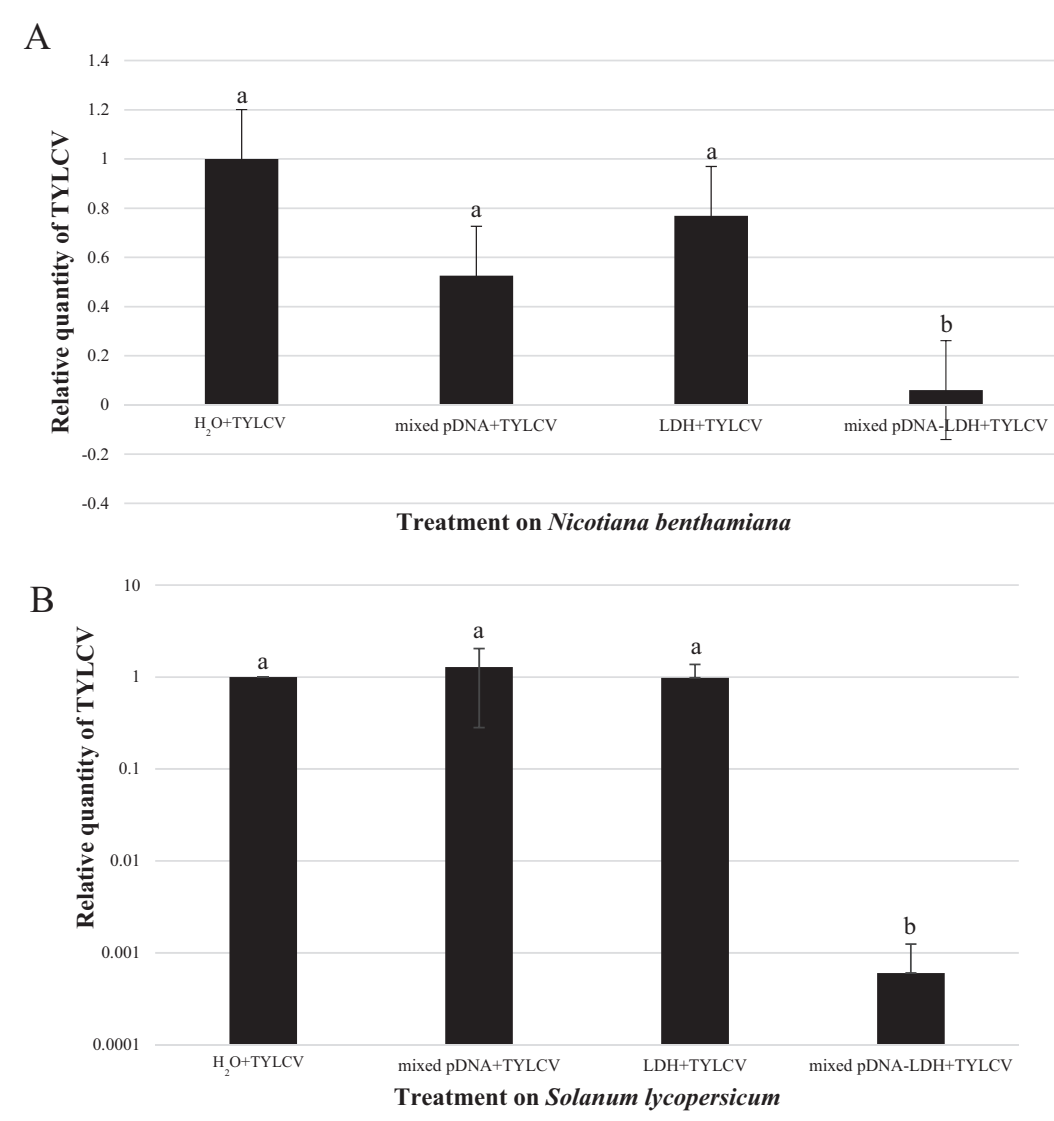

Fig. 6 Quantities of tomato yellow leaf curl virus (TYLCV) for diverse treatments. Quantities of tomato yellow leaf curl virus (TYLCV) in leaves of Nicotiana benthamiana (a) and Solanum lycopersicum (b) at 35 days post inoculation detected using RT-PCR. TYLCV was inoculated 7 days after foliar application with either $\mathrm{H} 2 \mathrm{O}$, mixed pDNA (mixed with pDNA1, pDNA2, and pDNA3), layered double hydroxide (LDH) nanosheets, or mixed pDNA-LDH (mixed with pDNA1-LDH, pDNA2-LDH, and pDNA3-LDH). Each treatment had 60 replicates. Leaf DNA was extracted from mixed leaves collected from the top, middle, and bottom of each plant. The bars on each column represent standard deviations. Values (means \pm SEs) are the averages of three independent experiments, and significant differences $(P \leq 0.05)$ among treatments in each group are indicated by different letters

between hydroxide layers by a simple ion-exchange reaction to form bio-LDH nanohybrids with gentle orbital agitation, which is the simplest method of nanocomplex formation ${ }^{8,25,27,35}$. It is a simple, effective, easy-tofollow method for the large-scale application of pDNAs, and it exhibits slow release and does not raise biosafety concerns.

In generating amiRNAs, the choice of target site was crucial in amiRNA-mediated virus resistance ${ }^{36,37}$. Fortunately, it is relatively easy to pick the ideal amiRNA sequences targeting mRNAs without disturbing the expression of other genes. This feature enhances the robustness of this technology ${ }^{6}$. The application of multiple amiRNAs targeting the AV1/AV2 gene of TYLCV resulted in a high level of viral suppression over a certain period of time in this study (Fig. 4). This is thematically sound and promising in practice. This result is supported by results from many other studies. These studies documented effects of viral diseases such as tomato leaf curl
New Delhi virus ${ }^{37,38}$, TYLCV-Israel ${ }^{39}$, tomato leaf curl Java virus ${ }^{40}$, cotton leaf curl Burewala virus ${ }^{41}$, and bean golden mosaic virus ${ }^{42}$. Multiple resistance mediated by a single pre-amiRNA was often designed using the conserved sequence of virus genes to inhibit multiple virus strains simultaneously ${ }^{43-45}$. In addition, multiple preamiRNAs concatenated under the same promoter can express multiple amiRNAs and mediate multivirus resistance in plants ${ }^{46,47}$. These antiviral strategies mediated by amiRNA were simple and feasible in specific experimental operations. Aragao demonstrated the feasibility of genetically engineered Geminivirus-resistant plants ${ }^{42}$. They used RNAi constructs to silence viral genes. This resulted in high resistance to viral infection, with approximately $93 \%$ of the plants being symptom free. It is important that LDH-mediated amiRNA is delivered into plants before viral infection for successful resistance. LDH nanomaterials can protect and slow the release of pDNA on the leaves for a period of time. Most likely, 
nanoparticle delivery in the plant cells was accomplished via natural openings-e.g., stomata (microscopic pores in leaf surfaces), hydathodes (stomata-like openings that secrete water), or plasmodesmata, which was basically consistent with the results reported by many other researchers ${ }^{8,20}$.

In conclusion, LDH-based pDNA is a promising tool for enhancing plant resistance against TYLCV. This method is worthy of further investigation for thorough exploitation of host-induced gene silencing against geminiviruses in future work.

\section{Materials and methods}

\section{Designing and cloning of amiRNA}

A schematic of the procedure for using pDNA-LDH to protect plants against Begomovirus is shown in Fig. S7. Tomato samples were collected in Henan Province of China. Polymerase chain reaction (PCR) was conducted using PA/PB primers and TY-FAN (+)/TY-FAN (-) reverse primers (Table S4). Three isolates of TYLCV were detected by comparing reference strains on the National Center for Biotechnology Information (NCBI, https:// www.ncbi.nlm.nih.gov/) (accession numbers MF668139, MF668140, and MF668141, respectively). Sequences of the CP gene (AV1/AV2) of the three TYLCV isolates were used as query objects for the BLAST algorithm, and conserved regions containing more than 21 nucleotides were identified as target sequences. The WMD3 program (http://wmd3.weigelworld.org/cgi-bin/webapp.cgi) was used to design three corresponding amiRNAs: amiR-AV1/ AV2-1 (5'-TATTATATCGCCTCGTCGCTT-3'), targeting the upstream region (7-27 bp) of the AV1 gene and the middle region $(167-187 \mathrm{bp})$ of the AV2 gene; amiRAV1-2 (5'-TTAACACAGAACCACTTACCC-3'), targeting the middle region of the AV1 gene (318-338 bp); and amiR-AV1-3 (5'-TCATATACAATAACGAGGCGT-3'), targeting the downstream region of the AV1 gene (680-700 bp).

Natural pre-miRNA (ath-miR-159a) was used as a backbone to generate three artificial pre-miRNAs (preamiRNAs). The sequences of pre-miR159a were replaced by those of the amiRNAs, and the whole precursors with the amiRNA sequence were synthesized and cloned into pMD19-T (simple) via $X b a$ I and Sac I enzyme cutting sites by Sangon Biotech (Shanghai, China). Subsequently, these sequences were cut from pMD19-T (simple) with $X b a$ I and $S a c$ I digestion enzymes and finally transferred into the binary vector pBI121 (also cut by $X b a$ I and $S a c$ I) via ligation by T4 DNA ligase. Expression of all the preamiRNAs was driven by the CaMV $35 \mathrm{~S}$ promoter and terminated with the NOS terminator. The clones were confirmed by DNA sequencing. Thus, three pre-amiRNAs, each containing amiR-AV1/AV2-1 (amir159-AV1/ AV2-1), amiR-AV1-2 (amir159-AV1-2), or amiR-AV1-3
(amir159-AV1-3), were cloned into the pBI121 vector and used in this study. Possible folding structures of the predicted transcripts from the pre-amiRNAs were determined using the mfold program (http://mfold.rna.albany. edu/?q=mfold/RNA-Folding-Form).

\section{Preparation of pDNA-LDH nanosheets}

LDH nanoparticles were made up of positively charged brucite-like layers, which were synthesized by a microwave-assisted aqueous coprecipitation method ${ }^{24,48}$. The approximate chemical formula of LDH nanomaterials was determined by energy-dispersive X-ray spectrometry (EDX-720, Shimadzu, Japan). LDH nanoparticles in suspension were characterized for size distribution and zeta potential using a Nanosizer Nano ZS (Malvern Panalytical Ltd, Malvern, UK). Morphological images of the particles were captured by TEM using a JEM-1400Plus instrument (acceleration voltage of $120 \mathrm{kV}$, JEOL, Tokyo, Japan) and a ZEISS Merlin scanning electron microscope (SEM, Carl Zeiss SMT AG, Oberkochen, Germany). White pellet-like powder was yielded by freeze-drying a concentrated suspension. The powdery product was analyzed with FTIR spectroscopy using a Brucker TENSOR 27 instrument scanning from 4000 to $400 \mathrm{~cm}^{-1}$ and powder X-ray diffraction using a PANalytical X'pert3 Powder X-ray diffractometer (slit width DS $=1 / 4^{\circ}$, scanning rate: $5^{\circ} \mathrm{min}^{-1}$, $2 \theta=5^{\circ}-80^{\circ}$, Co $\mathrm{K} \alpha$ radiation wavelength of $0.15418 \mathrm{~nm}$ ) to verify the chemical composition and crystal structure of the nanoparticles.

pDNAs of the recombinant pBI121 vector were isolated and purified with a Qiagen Plasmid Purification Kit (Qiagen, Beijing, China). With gentle orbital agitation, three pDNAs were loaded on LDH nanosheets by electrostatic interaction. To define optimal and complete loading of pDNA into LDH nanosheets, samples were prepared whose ratios of in vitro transcribed controlpDNA (500 ng) and pDNA ( $1 \mu \mathrm{g})$ to LDH (pDNA-LDH $(\mathrm{w} / \mathrm{w}))$ were $1: 1,1: 2,1: 4,1: 8,1: 10,1: 12$, and 1:16. A $10 \mu \mathrm{l}$ aliquot of pDNA and different proportions of $\mathrm{LDH}$ nanosheets were incubated at room temperature $\left(25^{\circ} \mathrm{C}\right)$ for $30 \mathrm{~min}$ with gentle orbital agitation. Complete pDNA loading was assessed by retention of pDNA-LDH complexes in wells of a $1 \%$ agarose gel. Three pDNA-LDH nanosheets were separately prepared, and the morphologies were imaged with TEM.

\section{Stability of pDNA on LDH nanosheets}

To examine the stability of pDNA loaded on LDH nanosheets, acidic and enzymatic treatments were applied. $\mathrm{pH}$ changes were monitored by dissolving LDH nanosheets under strongly acidic conditions by adding $5 \mathrm{ml}$ of $2 \mathrm{mM} \mathrm{HNO}_{3}$ (pH 3.0) to $5 \mathrm{ml}$ of $1 \mu \mathrm{g} / \mu \mathrm{l} \mathrm{LDH}$ nanosheets. $\mathrm{pH}$ values were measured at $1,3,10,20,30$, and $60 \mathrm{~min}$ after treatment. The release of pDNA from 
the pDNA-LDH complexes was determined at different $\mathrm{pH}$ values. Control-pDNA-LDH $(1: 10,10 \mu \mathrm{l})$ was first precipitated by adding $30 \mu \mathrm{l}$ of $1 \mathrm{M} \mathrm{NaCl}$ for $20 \mathrm{~min}$. The precipitate was pelleted by centrifugation at $9000 \times g$ for $30 \mathrm{~min}$. Pellets were resuspended in $10 \mu \mathrm{l}$ solutions $(\mathrm{pH}$ 2.0-12.0, adjusted with either $\mathrm{HNO}_{3}$ or $\mathrm{NaOH}$ ) and subjected to $1 \%$ agarose gel electrophoresis within $5 \mathrm{~min}$ of resuspension. Since LDH nanosheet-bound pDNA did not migrate, fluorescence could be observed in the well.

The ability of LDH nanosheets to protect loaded pDNA from potential environmental breakdown was investigated by the exposure of pDNA (500 ng) and pDNA-LDH $(500 \mathrm{ng} / 2.5 \mu \mathrm{g})$ to DNase I (TaKaRa, Dalian, China) treatments. Samples were treated with $70 \mathrm{U}$ of DNase I for 4 min at $25^{\circ} \mathrm{C}$. pDNA was released by acidic dissolution of $\mathrm{LDH}$ nanosheets using release buffer $(4.11 \mathrm{ml}$ of $0.2 \mathrm{M}$ $\mathrm{Na}_{2} \mathrm{HPO}_{4}+15.89 \mathrm{ml}$ of $0.1 \mathrm{M}$ citric acid; $\mathrm{pH} 3$ ) at a pDNA-LDH buffer volume ratio of 5:1. Nuclease-treated and untreated samples were examined on a $1 \%$ agarose gel.

\section{Uptake and transfection of pDNA in plant cells}

To detect pDNA uptake by plants and consequent movement in plant cells, three pDNAs were labeled with $1,1 \mathrm{P}-(4,4,8,8$, tetramethyl-4,8-diazaundecamethylene) bis [4- [3-methyl-benzo-1,3-oxazol-2-yl] methylidene]-1,4dihydroquinolinium] tetraiodide (YOYO-1) (Invitrogen, Shanghai, China), a green fluorescent dye for visualizing DNA molecules, per the manufacturer's recommendations and then loaded on LDH nanosheets. The labeled pDNA-LDH nanosheets were sprayed on the inner surface of onion and the leaf surface of $N$. benthamiana. To visualize nuclei, epidermis of onion and leaves of $N$. benthamiana were stained with 4',6-diamidino-2-phenylindole (DAPI, $5 \mathrm{mg} / \mathrm{mL}$, Sigma-Aldrich, St. Louis, USA). The plant tissues were soaked in phosphate buffer solution (PBS) containing DAPI (pH 7.0; DAPI:PBS (v/v) = 1:1000) and kept in darkness for $20 \mathrm{~min}$. The soaked tissues were washed with PBS three times before observation. The samples were examined under a Zeiss LSM780 META confocal laser scanning microscope (Carl Zeiss, Jena, Germany) after $72 \mathrm{~h}$.

\section{amiRNA expression analysis}

$N$. benthamiana and S. lycopersicum "Moneymaker" were used as hosts for amiRNA expression. All plants were grown in potting soil in $10 \mathrm{~cm}$-wide pots under greenhouse conditions $\left(25^{\circ} \mathrm{C}, 70 \%\right.$ relative humidity) unless otherwise specified. At the three-leaf stage for $N$. benthamiana and two-leaf stage for S. lycopersicum, plants were sprayed with pBI121-LDH, pDNA1-LDH (amiRNA-AV1/AV2-1), pDNA2-LDH (amiRNA-AV1-2), pDNA3-LDH (amiRNA-AV1-3), or pDNA $(1+2+3)$ LDH (mixed with three pDNA-LDHs). The loading ratio of the five pDNA-LDH nanosheets was 1:10. Plants were sprayed with an atomizer at $\sim 125 \mu \mathrm{l} / \mathrm{cm}^{2}$ leaf surface.

qRT-PCR assays were performed to evaluate the expression levels of mixed mature miRNAs as previously described after 7 days. Total RNA and small RNA fractions of leaves of $N$. benthamiana and S. lycopersicum were extracted using a TRIzol (Life Technologies, USA)based method following the manufacturer's protocol. Expression of mixed miRNAs was quantified by qPCR using a Mir- $\mathrm{X}^{\mathrm{TM}}$ miRNA First-Strand Synthesis kit (TaKaRa Bio, USA), which is a complete, dual function system for performing first-strand cDNA synthesis and qPCR to precisely measure the level of miRNAs. The three upstream primers were miRNA-specific primers, which are described in Table S4. The downstream primers were the mRQ $3^{\prime}$ primer provided in the kit. The results were normalized to the U6 gene of $N$. benthamiana and $S$. lycopersicum (the primer sequences amplifying the U6 gene are shown in Table S4). Mixed samples were used in qRT-PCR analysis, and three biologically independent replicates were analyzed for each sample. Plants sprayed with pBI121-LDH were used as controls. Data were analyzed using the $2^{-\Delta \Delta \mathrm{Ct}}$ method.

\section{Foliar spray with pDNA-LDH for TYLCV prevention Plant inoculation with TYLCV}

$N$. benthamiana and S. lycopersicum "Moneymaker" were used as hosts for symptom expression. At the threeleaf stage for $N$. benthamiana and two-leaf stage for $S$. lycopersicum, plants were sprayed with either $\mathrm{H}_{2} \mathrm{O}$ (distillation-distillation $\mathrm{H}_{2} \mathrm{O}$ ), LDH, $\mathrm{pDNA}(1+2+3$ ) (mixture of three pDNAs), or $\mathrm{pDNA}(1+2+3)-\mathrm{LDH}$. The loading ratio of pDNA1, pDNA2, and pDNA3 to nanosheets in the pDNA-LDH complex was $1: 10 . \mathrm{H}_{2} \mathrm{O}$, pDNA (1:0), and LDH (0:3) were used as controls. The leaf surfaces of plants were sprayed with $1.25 \mu \mathrm{g}$ of pDNA, $3.75 \mu \mathrm{g}$ of $\mathrm{LDH}$, or $\mathrm{H}_{2} \mathrm{O}$ using an atomizer at $\sim 125 \mu \mathrm{l} /$ $\mathrm{cm}^{2}$. Leaf cells and stomates of $N$. benthamiana and $S$. lycopersicum plants were observed $30 \mathrm{~min}$ after foliar spraying to evaluate the effects of treatment. Seven days later, viral challenging assays were carried out in the sprayed plants using an agro-infectious clone of TYLCV[CN:SH2] (GenBank accession number: AM282874). Plants were kept at $25^{\circ} \mathrm{C}$ with $70 \%$ relative humidity and a $16 \mathrm{~h} / 8 \mathrm{~h}$ photoperiod in an insect-free greenhouse, and symptom development was examined daily.

\section{Detection of $\mathrm{H} 2 \mathrm{O} 2$ in plants}

TYLCV-inoculated leaves were subjected to 3,3-diaminobenzidine (DAB) staining ${ }^{49}$ to investigate the accumulation of $\mathrm{H}_{2} \mathrm{O}_{2}$ and its production location. Leaves of $N$. benthamiana and S. lycopersicum were excised from the plant and placed in $20 \mathrm{~mL}$ tubes, covered with $\mathrm{DAB}-\mathrm{HCl}$ solution $(1 \mathrm{mg} / \mathrm{ml}, \mathrm{pH} 3.8)$ and incubated in a growth 
chamber for $8 \mathrm{~h}$ at $25^{\circ} \mathrm{C}$. Once red-brown DAB solution moved up to the top of leaves along the veins, chlorophyll from whole leaves was removed by directly immersion into a fixing solution (anhydrous ethanol:acetic acid $=3: 1$ ) for $24 \mathrm{~h}$. The stained leaves were processed with hydrated trichloroacetaldehyde three times and observed using an optical microscope.

\section{Delivery of pDNA}

To determine whether pDNAs were delivered into plant leaves, PCR was conducted on unsprayed new leaf samples of both $N$. benthamiana and S. lycopersicum. Total DNA of leaves was extracted using a plant genomic DNA extraction kit (TaKaRa, Dalian, China) following the manufacturer's protocol. The primers pBI121-F/pBI121-R (Table S4) were used to amplify the pBI121 vector.

\section{Detection of TYLCV using $q P C R$}

Expression of TYLCV was quantified by targeting leaves from mixed leaves collected from the top, middle, and bottom of plants by qPCR using SYBR Premix Ex Taq (TaKaRa) on an ABI Prism 7500 thermal cycler (Applied Biosystems, Waltham, USA) with the primer pairs $\mathrm{Nb}$ GAPDH-F/Nb-GAPDH-R, TYLCV-YG-3/TYLCV-YG-4, and Tomato25s-Rrna-UNIV (+)/Tomato25s-Rrna-UNIV (-) (Table S4). Triplicate PCRs were conducted for each DNA sample, and the assay consisted of three technical replicates. Data were analyzed using the $2^{-\Delta \Delta C t}$ method.

\section{Detection of TYLCV using ELISA analysis}

TYLCV was detected from mixed leaves collected from the top, middle and bottom of plants using antigen-coated plate (ACP)-ELISA according to standard methods. The antibody used in ACP-ELISA was reacted against the CP of TYLCV. The reactions were followed by measuring the optical density at $405 \mathrm{~nm}$ on a spectrum plate reader (Thermo Fisher Scientific, Waltham, USA). A sample was considered TYLCV positive when the absorbance values were two times higher than those of noninoculated plant samples.

\section{Acknowledgements}

This research was funded by the National Natural Science Foundation of China (31801707), the Key Projects of Science and Technology Research in Henan Province (182102110470), the Plant Protection of Key Discipline Project of Henan province (107020219001/005), the National Key Research and Development Program of China (2016YFD0300203-3), a First-Class Postdoctoral Research Grant in Henan Province (001701038), and the Science-Technology Foundation for High Level Talent of Henan Institute of Science and Technology (2015028). The authors gratefully acknowledge Prof. Xueping Zhou and Associate Researcher Xiuling Yang (Institute of Plant Protection, Chinese Academy of Agricultural Sciences, China) for providing TYLCV infectious clones and specific antibodies.

\section{Author details}

${ }^{1}$ Postdoctoral Research Base, Henan Institute of Science and Technology, Xinxiang, China. ${ }^{2}$ College of Plant Protection, Henan Agricultural University, Zhengzhou, Henan, China. ${ }^{3}$ Henan Engineering Research Center of Crop
Genome Editing, Henan Institute of Science and Technology, Xinxiang, China. ${ }^{4}$ State Key Laboratory of Pulp \& Paper Engineering, South China University of Technology, Guangzhou, China. ${ }^{5}$ Key Laboratory of Plant Genetics and Molecular Breeding, Zhoukou Normal University, Zhoukou, China. 'School of Food and Agriculture, The University of Maine, Orono, ME 04469, USA

\section{Author contributions}

Q.L.Liu designed and performed the research and wrote the paper. Y.P.Li, Q.Liu, and W.P.Wang performed the research. K.D.X., D.X.Li, P.W.S., and R.F.B. performed some tests of the experiments. Q.C.W., Y.A.Y., H.L.S., and H.Y.H. planted some samples for the experiments. C.W.Li, H.L.Li, and X.H.Wang designed the research. J.J.H. revised the paper.

\section{Conflict of interest}

The authors declare that they have no conflict of interest.

Supplementary Information accompanies this paper at (https://doi.org/ 10.1038/s41438-020-00400-2).

Received: 30 April 2020 Revised: 26 August 2020 Accepted: 1 September 2020

Published online: 01 November 2020

\section{References}

1. Scholthof, K. G. et al. Top 10 plant viruses in molecular plant pathology. Mol. Plant Pathol. 12, 938-954 (2011).

2. Zerbini, F. M. et al. ICTV virus taxonomy profiles: geminiviridae. J. Gen. Virol. 98 131-133 (2017).

3. Zaidi, S. S., Tashkandi, M., Mansoor, S. \& Mahfouz, M. M. Engineering plant immunity: using CRISPR/Cas9 to generate virus resistance. Front. Plant Sci. $\mathbf{7}$ 1-10 (2016).

4. Tiwari, M., Sharma, D. \& Trivedi, P. K. Artificial microRNA mediated gene silencing in plants: progress and perspectives. Plant Mol. Biol. 86, 1-18 (2014).

5. Warthmann, N. et al. Highly specific gene silencing by artificial miRNAs in rice. PLOS ONE 3, e1829 (2008).

6. Schwab, R. et al. Highly specific gene silencing by artificial microRNAs in Arabidopsis. Plant Cell 18, 1121-1133 (2006).

7. Khraiwesh, B. et al. Specific gene silencing by artificial microRNAs in Physcomitrella patens: an alternative to targeted gene knockouts. Plant Physiol. 148, 684-693 (2008).

8. Cunningham, F. J. et al. Nanoparticle-mediated delivery towards advancing plant genetic engineering. Trends Biotechnol. 36, 882-897 (2018).

9. Pudake, R. N., Kumari, M., Sahu, B. B. \& Sultan, E. Targeted gene disruption tools for fungal genomics. In Modern Tools and Techniques to Understand Microbes (eds. Varma A., Sharma A. K.) 81-102. (Springer-Verlag, 2017).

10. Nyaboga, E., Tripathi, J. N., Manoharan, R. \& Tripathi, L. Agrobacteriummediated genetic transformation of yam (Dioscorea rotundata): an important tool for functional study of genes and crop improvement. Front. Plant Sci. 5, 463 (2014).

11. Han, Y., Blechl, A. \& Wang, D. The distribution of cotransformed transgenes in particle bombardment-mediated transformed wheat. Transgenic Res. 24, 1055-1063 (2015).

12. Gelvin, S. B. Integration of agrobacterium T-DNA into the plant genome. Annu. Rev. Genet. 51, 195-217 (2017).

13. Stoddard, T., Baltes, N. J. \& Luo, S. Agrobacterium-mediated genome modification without T-DNA integration. U.S. patent application 15/013,323[P] (2016).

14. Tan, L. \& Sun, X. Recent advances in mRNA vaccine delivery. Nano Res. 11 5338-5354 (2018).

15. Eapen, S. \& D'Souza, S. F. Prospects of genetic engineering of plants for phytoremediation of toxic metals. Biotechnol. Adv. 23, 97-114 (2005).

16. Kuzma, J. Moving forward responsibly: oversight for the nanotechnologybiology interface. J. Nanoparticle Res. 9, 165-182 (2006).

17. Mukhopadhyay, S. S. Nanotechnology in agriculture: prospects and constraints. Nanotechnol. Sci. Appl. 7, 63-71 (2014).

18. Yuan, Z. et al. Novel impacts of functionalized multi-walled carbon nanotubes in plants: promotion of nodulation and nitrogenase activity in rhizobiumlegume system. Nanoscale 9, 9921-9937 (2017). 
19. Agency, U. E. P. Nanotechnology white paper. Report EPA 100/B-07/001. http://www.epa.gov/osainter/pdfs/nanotech/epananotechnologywhitepaper-0207.pdf (2014).

20. Mitter, N. et al. Clay nanosheets for topical delivery of RNAi for sustained protection against plant viruses. Nat. Plants 3, 16207 (2017).

21. Jiang, L. et al. Systemic gene silencing in plants triggered by fluorescent nanoparticle-delivered double-stranded RNA. Nanoscale 6, 9965-9969 (2014).

22. Li, H., Guan, R., Guo, H. \& Miao, X. New insights into an RNAi approach for plant defence against piercing-sucking and stem-borer insect pests. Plant Cell Environ. 38, 2277-2285 (2015).

23. Lau, S., Schwarzacher, T., Othman, R. Y. \& Harikrishna, J. A. dsRNA silencing of an R2R3-MYB transcription factor affects flower cell shape in a Dendrobium hybrid. BMC Plant Biol. 15, 194-207 (2015).

24. Xu, Z. P. et al. Stable suspension of layered double hydroxide nanoparticles in aqueous solution. J. Am. Chem. Soc. 128, 36-37 (2006).

25. Ladewig, K., Xu, Z. P. \& Lu, G. Q. M. Layered double hydroxide nanoparticles in gene and drug delivery. Expert Opin. Drug Del. 6, 907-922 (2009).

26. Kim, H., Shimura, H. \& Masuta, C. Advancing toward commercial application of RNA silencing-based strategies to protect plants from viral diseases. J. Gen. Plant Pathol. 85, 1-8 (2019).

27. Choy, J. H., Kwak, S. Y., Jeong, Y. J. \& Park, J. S. Inorganic layered double hydroxides as nonviral vectors. Angew. Chem. Int Ed. 39, 4042-4045 (2000).

28. Tyner, K. M. et al. Intercalation, delivery, and expression of the gene encoding green fluorescence protein utilizing nanobiohybrids. J. Control. Release $\mathbf{1 0 0}$ 399-409 (2004).

29. Desigaux, L. et al. Self-assembly and characterization of layered double hydroxide/DNA hybrids. Nano Lett. 6, 199-204 (2006).

30. Ladewig, K. Nanoparticles with Application in the Delivery of Nucleic Acids to Mammalian Cells Ph.D. Dissertation, (The University of Queensland, Queensland, 2009).

31. Holmes, D. S. \& Quigley, M. A rapid boiling method for the preparation of bacterialplasmids. Anal. Bioehem. 114, 193-197 (1981).

32. Gómez-Márquez, J., Freire, M. \& Segade, F. A simple procedure for large-scale purification of plasmid DNA. Gene 54, 255 (1987).

33. HC, B. \& J, D. A rapid alkaline extraction procedure for screening recombinant plasmid DNA. Nucleic Acids Res. 7, 1513-1523 (1979).

34. Wright, J. L., Jordan, M. \& Wurm, F. M. Extraction of plasmid DNA using reactor scale alkaline lysis and selective precipitation for scalable transient transfection. Cytotechnology 35, 165-173 (2001).

35. Wang, $\mathrm{H}$. et al. Cationic starch/pDNA nanocomplexes assembly and their nanostructure changes on gene transfection efficiency. Sci. Rep. 7, 14844 (2017).
36. Jiang, F. et al. The choice of target site is crucial in artificial miRNA-mediated virus resistance in transgenic Nicotiana tabacum. Physiol. Mol. Plant Pathol. 76 2-8 (2011).

37. Vu, T. V., Roy Choudhury, N. \& Mukherjee, S. K. Transgenic tomato plants expressing artificial microRNAs for silencing the pre-coat and coat proteins of a begomovirus, Tomato leaf curl New Delhi virus, show tolerance to virus infection. Virus Res. 172, 35-45 (2013).

38. Singh, A., Taneja, J., Dasgupta, I. \& Mukherjee, S. K. Development of plants resistant to tomato geminiviruses using artificial trans-acting small interfering RNA. Mol. Plant Pathol. 16, 724-734 (2015).

39. Zrachya, A. et al. Suppressor of RNA silencing encoded by Tomato yellow leaf curl virus-Israel. Virology 358, 159-165 (2007).

40. Sharma, P. \& Ikegami, M. Tomato leaf curl Java virus V2 protein is a determinant of virulence, hypersensitive response and suppression of posttranscriptional gene silencing. Virology 396, 85-93 (2010).

41. Ali, I., Amin, I., Briddon, R. W. \& Mansoor, S. Artificial microRNA-mediated resistance against the monopartite begomovirus Cotton leaf curl Burewala virus. Virol. J. 10, 231 (2013).

42. Aragao, F. J. \& Faria, J. C. First transgenic geminivirus-resistant plant in the field. Nat. Biotechnol. 27, 1086-1088 (2009). author reply 1088-9.

43. Zhang, $X$. et al. Expression of artificial microRNAs in tomato confers efficient and stable virus resistance in a cell-autonomous manner. Transgenic Res. 20, 569-581 (2011)

44. Kung, Y. et al. Multiple artificial microRNAs targeting conserved motifs of the replicase gene confer robust transgenic resistance to negative-sense singlestranded RNA plant virus. Mol. Plant Pathol. 13, 303-317 (2012)

45. Song, Y. et al. Effects of the sequence characteristics of miRNAs on multi-viral resistance mediated by single amiRNAs in transgenic tobacco. Plant Physiol. Biochem. 77, 90-98 (2014).

46. $\mathrm{Ai}$, $\mathrm{T}$. et al. Highly efficient virus resistance mediated by artificial microRNAs that target the suppressor of PVX and PVY in plants. Plant Biol. (Stuttg.) 13 304-316 (2011).

47. Fahim, M., Millar, A. A., Wood, C. C. \& Larkin, P. J. Resistance to Wheat streak mosaic virus generated by expression of an artificial polycistronic microRNA in wheat. Plant Biotechnol. J. 10, 150-163 (2012).

48. Dong, $\mathrm{H}$. et al. Engineering small MgAl-layered double hydroxide nanoparticles for enhanced gene delivery. Appl. Clay Sci. 100, 66-75 (2014).

49. Huckelhoven, R., Fodor, J., Preis, C. \& Kogel, K. H. Hypersensitive cell death and papilla formation in barley attacked by the powdery mildew fungus are associated with hydrogen peroxide but not with salicylic acid accumulation Plant Physiol. 119, 1251-1260 (1999). 